\title{
Impact of Active Breathing Control-Deep Inspiration Breath Hold (ABC-DIBH) on the dose to surrounding normal structures in tangential field left breast radiotherapy
}

\author{
Chia-Chuan Kuo ${ }^{1}$, Chin-Chieh Chang ${ }^{1}$, Hao-Wen Cheng ${ }^{1}$, Jo-Ting Tsai ${ }^{1,2}$ \\ ${ }^{1}$ Department of Radiation Oncology, Shuang Ho Hospital, Taipei Medical University, Taipei, Taiwan; ${ }^{2}$ Department of Radiology, School of \\ Medicine, College of Medicine, Taipei Medical University, Taipei, Taiwan \\ Contributions: (I) Conception and design: CC Kuo, JT Tsai; (II) Administrative support: JT Tsai; (III) Provision of study material or patients: JT Tsai; \\ (IV) Collection and assembly of data: CC Kuo, CC Chang; (V) Data analysis and interpretation: CC Kuo, CC Chang; (VI) Manuscript writing: All \\ authors; (VII) Final approval of manuscript: All authors. \\ Correspondence to: Jo-Ting Tsai, MD, PhD. Department of Radiation Oncology, Shuang Ho Hospital, Taipei Medical University, No. 291, \\ Zhongzheng Rd., Zhonghe Dist., New Taipei City 235, Taiwan. Email: 10576@s.tmu.edu.tw.
}

Background: Adjuvant radiotherapy after breast conserving surgery (BCS) is considered standard of care. For greater homogeneity of target dose and better normal tissue sparing, several radiotherapy modalities evolved. Therefore, we aimed to evaluate the organ-sparing potential of deep inspiration breath hold (DIBH) technique by comparing with the treatment plans among different respiratory phases based on the images retrieved from deep breathing 4D-CT.

Methods: This retrospective study was conducted from August 2017 to February 2019, and included 14 patients with left-sided breast cancer who received adjuvant radiotherapy after BCS. Two sets of CT scans were performed, including 3D-CT images of DIBH with the active breathing control (ABC) device, and deep breathing 4D-CT images. We used tangential angle IMRT plans with four portals for treatment planning. Three plans were generated for each patient. One plan was based on the 3D-CT images of DIBH, and the others were based on the 4D-CT images of middle of lung expansion (MLE) and start of lung expansion (SLE). Adjuvant radiotherapy was prescribed with a total radiation dose of $50 \mathrm{~Gy}$ in 25 fractions.

Results: The mean heart dose was 4.01 Gy in SLE, 3.76 Gy in MLE, and 2.72 Gy in DIBH (P<0.001). The plans with DIBH showed significantly lower dose for the heart, left ventricle (LV), left anterior descending coronary artery (LAD), and left lung when compared with the plans of either MLE or SLE images $(\mathrm{P}<0.05)$. Through inhalation cycle, the irradiated dose to heart and LV gradually decreased. There was no significant difference in right lung mean dose and $\mathrm{V} 5_{\text {right lung }}(\mathrm{P}>0.05)$.

Conclusions: For the adjuvant treatment of breast cancer, the use of DIBH technique resulted in a significant dose reduction in heart, LV, LAD, and left lung, especially with enough deep inhalation. Hence, the DIBH technique could be considered as a promising tool for normal organ sparing.

Keywords: Breast cancer; Deep Inspiration Breath Hold (DIBH); Active Breathing Control (ABC); radiotherapy

Received: 02 July 2020; Accepted: 28 September 2020; Published: 30 December 2020.

doi: $10.21037 /$ tro-20-49

View this article at: http://dx.doi.org/10.21037/tro-20-49

\section{Introduction}

Breast cancer is the most commonly diagnosed cancer and the leading cause of cancer death among women worldwide. It is estimated that about 2.1 million newly diagnosed female breast cancer cases in 2018, accounting for almost 1 in 4 cancer cases among women based on the report from the International Agency for Research on Cancer (IARC) (1). Specifically, breast cancer among Asian females has been 
associated with an early age-onset with a peak incidence at 45-49 years. This is in stark contrast to the age-specific pattern observed among US non-Hispanic white women with the highest incidence in the 75- to 79-year-old age group, 30 years older than for women in Asia (2).

In general, complete surgical resection is the primary treatment for breast cancer. Following operation, local treatment with radiotherapy and systemic treatment with chemotherapy, endocrine therapy, target therapy, or combination of these are used to reduce risk of recurrence. The need for multimodality therapies is based on several prognostic and predictive factors, including tumor histology, clinical stage, pathological characteristics, lymph node status, hormone receptor and human epidermal growth factor receptor 2 (HER2) status. In addition, patients' comorbidities and age should be taken into serious consideration as well (3). A meta-analysis by Early Breast Cancer Trialists' Collaborative Group (EBCTCG) was conducted to compare observation versus postoperative radiotherapy following breast conserving surgery (BCS). The study revealed that the 10 -year risk of locoregional or distant recurrence was as high as 35 present after BCS. Adjuvant radiotherapy to the conserved breast could not only halve the recurrent rate but also reduce the breast cancer death rate by about a sixth (4). Adjuvant whole breast radiotherapy with or without regional node irradiation after BCS is considered standard of care. Conventional fractionation with a dose of 46-50 Gy in 23-25 fractions is commonly used. However, acute or late skin reaction, cardiac toxicity, radiation pneumonitis, radiation fibrosis, second malignancies and rib fracture are possible complications of radiotherapy (5). Darby et al. has reported that after radiotherapy for breast cancer, the mean doses to the heart is 4.9 Gy in average; the rates of major coronary events which including myocardial infarction, coronary revascularization, or death from ischemic heart disease increases linearly with the mean dose to the heart by $7.4 \%$ per Gy. More importantly, there is no apparent threshold below which there is no risk (6). A recent systematic review reported that mean heart dose for left-sided breast cancer is lower (3.6 Gy) than previously reported (5.4 Gy), due to several advancement in RT technique (7).

Moreover, the reported frequency of radiation pneumonitis in breast cancer ranges from $1-80 \%$ due to variations in treatment techniques, total dose, use of photons/electrons, and use of various grading systems (8). A recent prospective study with two- to four-field radiotherapy for breast cancer reported that the overall incidence of pneumonitis was $13 \%(9)$.

For greater homogeneity of target dose and better normal tissue sparing, modern radiation techniques are used, including compensators such as tissue wedges, highly conformal treatment modalities such as intensity modulation radiation therapy (IMRT), tomotherapy and proton therapy. In addition, prone positioning and respiratory control techniques, including deep inspiration breath hold (DIBH) and respiratory gating, could be used to minimize the dose to lung and heart by pulling the breast and chest wall away from the heart. According to Bergom et al.'s review article, DIBH could significantly decrease the mean heart dose by $25-67 \%$ and mean LAD dose by $20-73 \%$, respectively compared with the same patients planned with free breathing (FB) and DIBH (10). Most studies compared FB with DIBH under three-dimensional computed tomography (3D-CT) for dose distribution. However, FB is only a random phase of patients' respiration, and could not reflect the actual volume of lung and heart which could be spared in the treatment field. Concerning for the surrounding healthy tissue, a comparison of the normal tissue sparing of DIBH technique should be elucidated.

Accordingly, the aim of this study was to investigate the dose distributions in the planning target volume (PTV) and organs at risk (OARs) according to the different respiratory phases. We evaluated the organ-sparing potential of DIBH technique by comparing with the treatment plans based on the images retrieved from deep breathing 4D-CT.

We present the following article in accordance with the MDAR reporting checklist (available at http://dx.doi. org/10.21037/tro-20-49).

\section{Methods}

\section{Patient data}

We enrolled twenty newly diagnosed patients with leftsided breast cancer who received adjuvant radiotherapy after breast conserving surgery from August 2017 to February 2019. Our patient database was reviewed, and the patients were identified as meeting the following criteria: (I) pathology-proven invasive carcinoma or ductal carcinoma in situ in patients that had received adjuvant radiotherapy after left-sided breast conserving surgery (BCS), (II) patients who received adjuvant radiotherapy using DIBH technique, (III) patients who received left whole breast irradiation only, (IV) Eastern Cooperative Oncology Group 


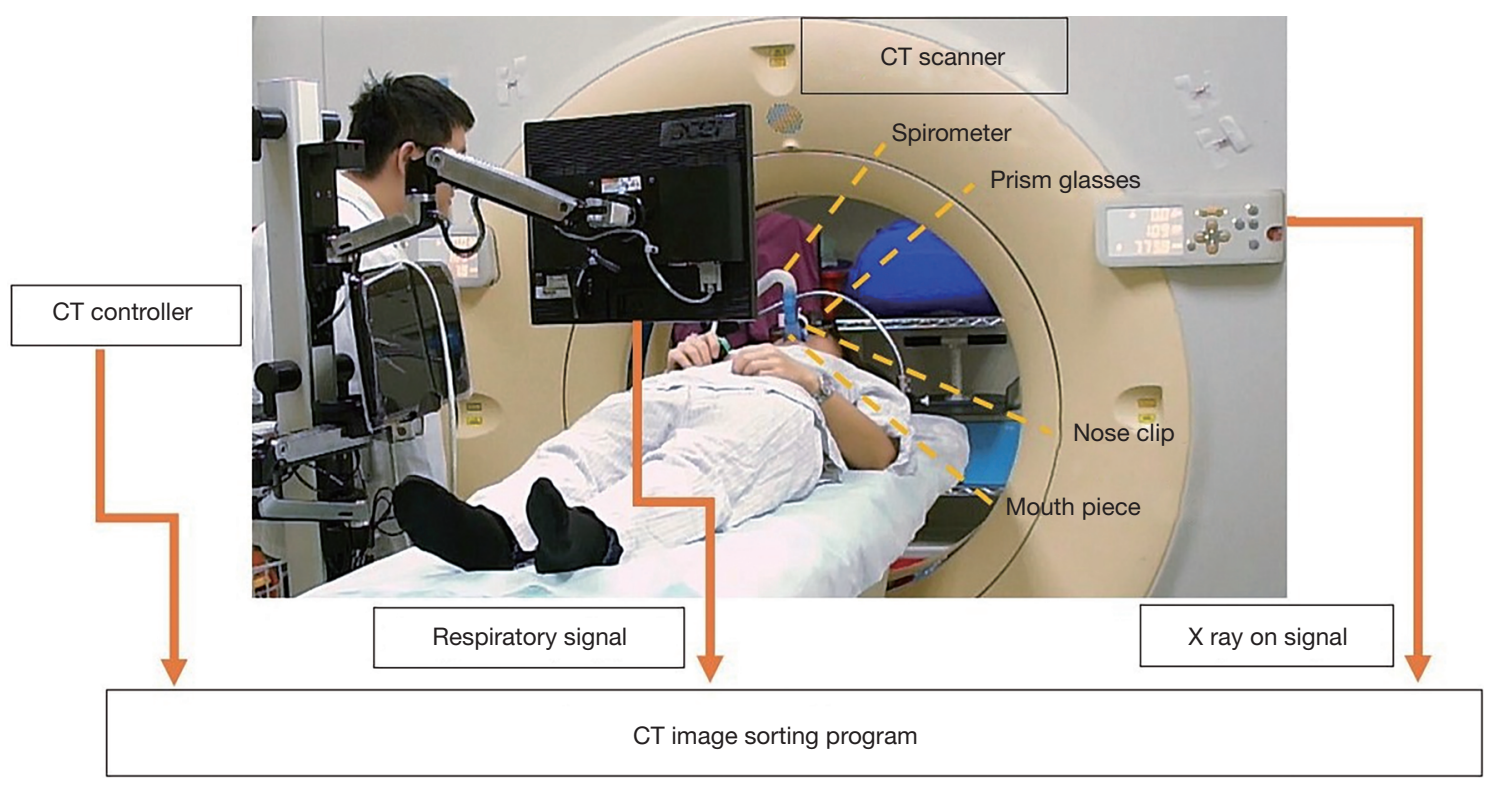

Figure 1 A demonstration of patient setup using DIBH technique with ABC device. DIBH, deep inspiration breath hold; ABC, active breathing control.

(ECOG) performance status 0. Exclusion criteria were: (I) patients who received previous chest wall surgery other than BCS, (II) patients who received previous breast or chest irradiation, (III) missing data or poor image quality. Tumors were staged according to the eighth edition of the American Joint Committee on Cancer (AJCC) staging system [2017].

The study was conducted in accordance with the Declaration of Helsinki (as revised in 2013). This study is retrospective, and approved by the hospital IRB (TMU-JIRB No.: N201912026) as "Expedited Review"; therefore, the informed consent is not required.

\section{CT simulation and DIBH techniques}

A Philips Brilliance Big Bore CT scanner (Philips Medical Systems, Cleveland, OH, USA) was used in this study to acquire CT images. All patients were immobilized in the supine position in a vacuum bag with their arms placed above their head. The DIBH technique was used for the patients who could hold their breath for 20 to 30 seconds. We performed DIBH technique with active breathing control (ABC) device (Elekta, Sweden). Patients were coached to breathe through their mouth by using a nose clip and a mouth piece connected to the device. The ABC device utilizes a spirometer which allows for monitoring of air flow throughout the respiratory cycle and stopping air flow at a set threshold volume (about $80 \%$ of maximum inspiratory capacity), causing patients to hold their breath to maintain this volume. The prism glasses enable patients to see their breathing cycle on the monitor attached to the $\mathrm{ABC}$ system. The patients inspire to a specified threshold and then hold that level of inspiration during every radiation therapy field delivered (Figure 1). In order to evaluate the dosimetric impact of ABC procedure, two sets of CT scans $(5 \mathrm{~mm}$ slice thickness) without contrast were performed for each patient from upper neck to mid-abdomen. 3D-CT images were obtained from the first DIBH CT scan with the ABC system, and 4D-CT images were obtained from the second deep-breathing CT scan (Figure 2).

For comparing the doses to OARs among different lung expansions, the patients were told to take deep inspiration to nearly the same level of DIBH and then to take deep inhalation for several cycles during 4D-CT scanning (Figure 3). A respiratory belt was used to record the amplitude and displacement of the abdominal motion and also the time interval. One respiratory wave was defined as the distance from the respiratory peak to the next. During the specific period of time that CT scanned the chest, usually there are three to six respiratory waves. A 4D-CT dataset was generally retrospective sorted with phase binning, and each respiratory cycle was divided into ten phases, from $0 \%$ to $90 \%$. Peak inhalation was set at 

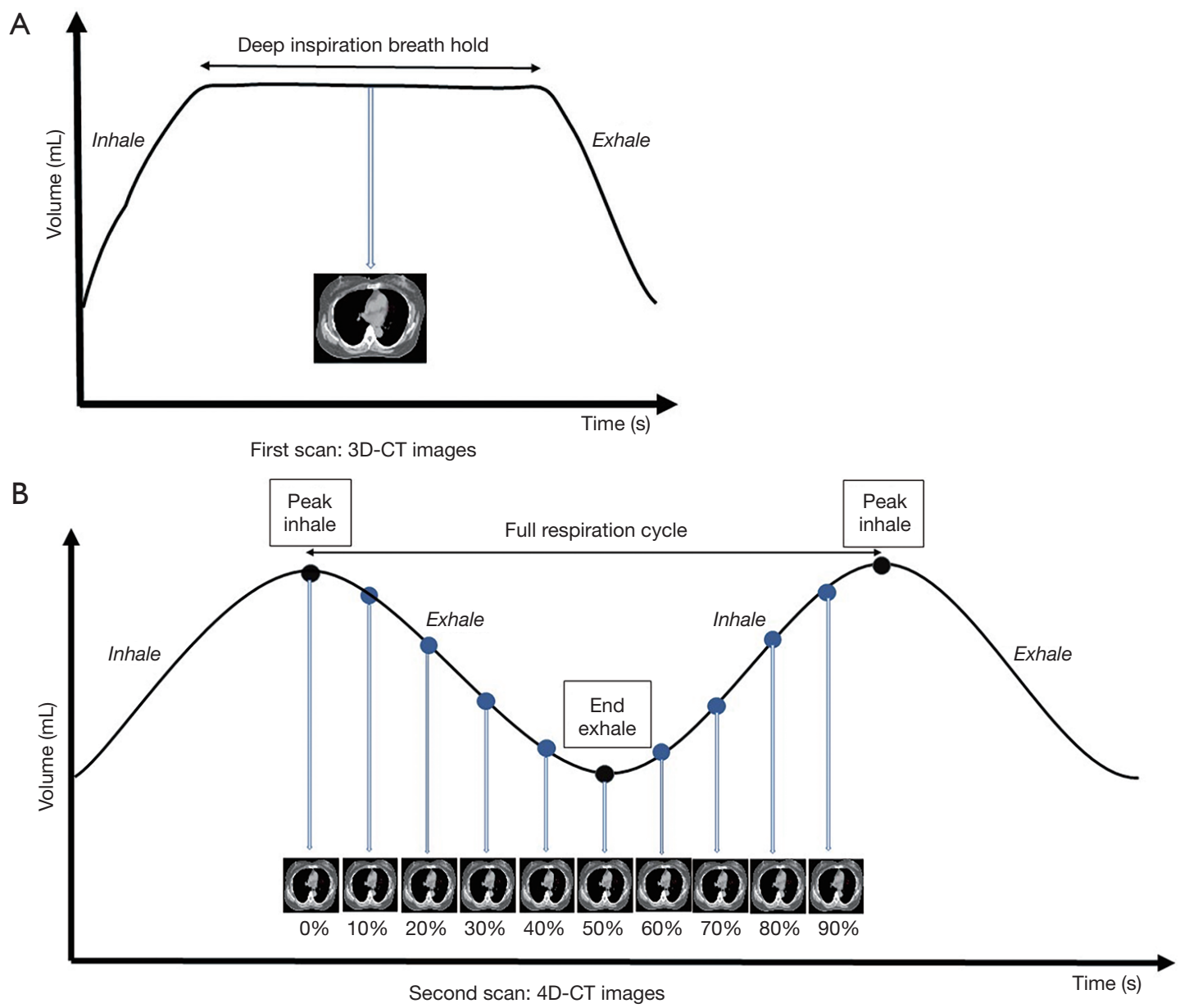

Figure 2 Overview of respiratory phase CT images. (A) First DIBH CT scan: 3D-CT images were obtained with the ABC system, (B) second deep-breathing CT scan: 4D-CT images were obtained. DIBH, deep inspiration breath hold; ABC, active breathing control.

$0 \%$ respiratory phase and end exhalation was set at $50 \%$ respiratory phase, as shown in Figure 2B. When designing the framework of these figures, we took previous studies regarding DIBH technique and $4 \mathrm{D}-\mathrm{CT}$ acquisition as reference (10-12). The images and data were all retrieved from our devices.

\section{Planning requirement and target delineation}

We used tangential angle IMRT plans with four portals with $6 \mathrm{MV}$ photon beams using Pinnacle Treatment Planning System Version 14 (Philips Radiation Oncology Systems, Fitchburg, WI) for treatment planning. The Adaptive Convolution algorithm with grid size $3 \mathrm{~mm}$ was used. Three IMRT plans were generated for each patient. One plan was based on the $3 \mathrm{D}-\mathrm{CT}$ images of DIBH with the ABC device, and the others were based on the 4D-CT images of middle of lung expansion (MLE) at 70\% respiratory phase and start of lung expansion (SLE) at 50\% respiratory phase (Figure 3).

The clinical target volume (CTV) was defined as the left whole breast according to the Radiation Therapy Oncology Group (RTOG) guidelines. The PTV was defined as the CTV with an isotropic expansion of a $5-\mathrm{mm}$ margin. The PTV_eval was created from the PTV cropped $3 \mathrm{~mm}$ from the skin surface for avoiding buildup effect which would cause high skin doses. The PTV_eval was the final target volume for planning $(13,14)$. In addition, considering the breathing motion during treatment, we added $1-\mathrm{cm}$ virtual bolus (no density was applied) outside the skin to achieve skin flash during optimization (low objective value for this region) $(15,16)$.

The heart, left ventricle (LV), left anterior descending coronary artery (LAD) and lung were delineated on each scan. The heart was contoured along with the pericardial 


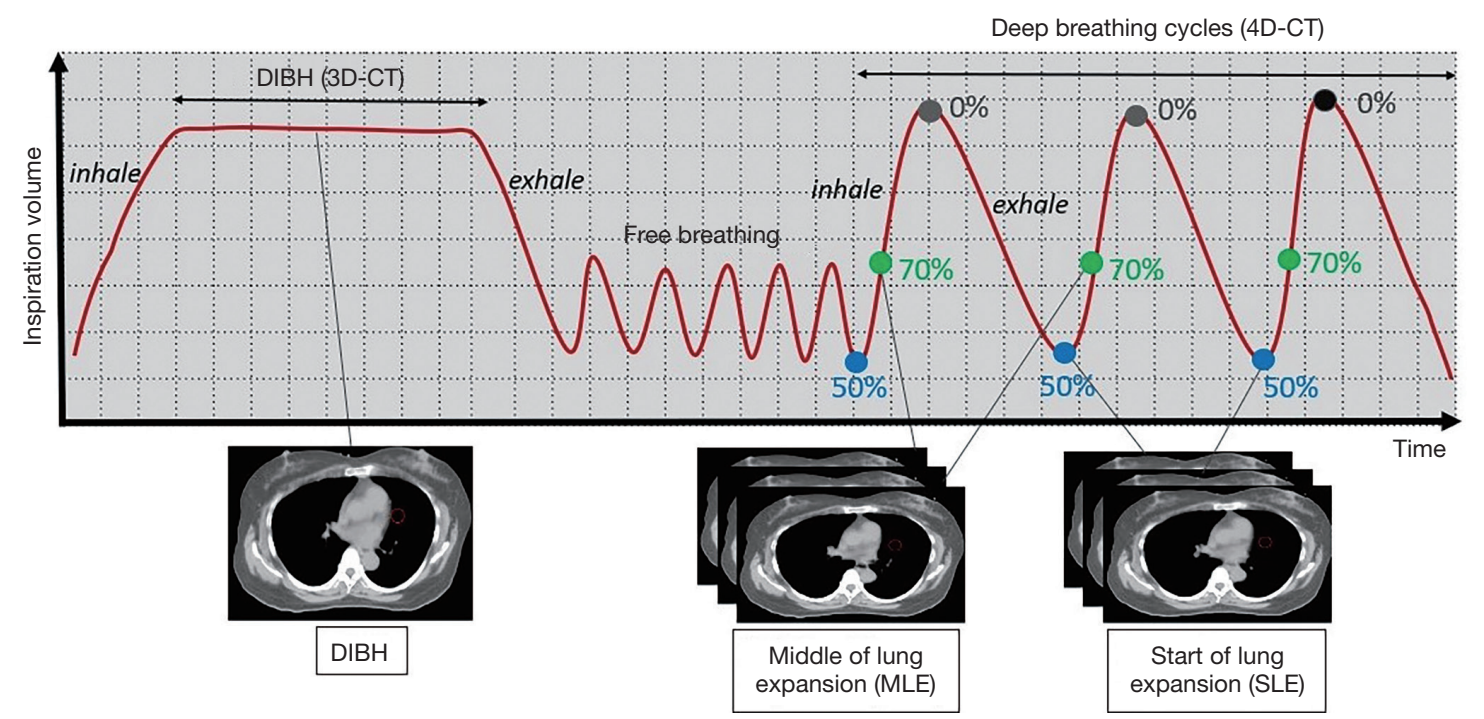

Figure 3 A demonstration of respiratory wave during CT simulation: 3D-CT images of DIBH with the ABC device, and 4D-CT images of MLE images at 70\% respiratory phase and SLE at 50\% respiratory phase. ABC, active breathing control; DIBH, deep inspiration breath hold; MLE, middle of lung expansion; SLE, start of lung expansion.

sac with the superior aspect began at the level of the inferior aspect of the pulmonary artery passing the midline and extended inferiorly to the apex of the heart. The LV and LAD were delineated following the heart atlas described by Feng et al. (17). The LAD artery was delineated from the origin of left coronary artery to the interventricular groove between the right and left ventricle. A total dose of $50 \mathrm{~Gy}$ in 25 fractions was prescribed to the PTV_eval. All plans were aimed to cover at least $95 \%$ of the PTV eval by $95 \%$ of the prescribed dose (V95\% $\geq 95 \%, \mathrm{Vx} \%$ : the percent volume receiving at least $\mathrm{x} \%$ of the prescribed dose), and the maximum dose (Dmax) less than $110 \%$ of the prescribed dose. The plan quality was evaluated by PTV_eval coverage, and the dose to heart and lung. The dose constraints for OARs used in optimization were based on the Quantitative Analyses of Normal Tissue Effects in the Clinic Summary (QUANTEC) guidelines (18). All the structures were delineated by the same radiation oncologist, and all the radiation plans were completed by a certified medical physicist with 10-year experience. Dose-volume parameters acquired from the plans of different breathing phases were compared with each other using statistical tests.

\section{Statistical analyses}

Statistical analyses were conducted using Statistical Package for Social Sciences (SPSS) 23 (IBM SPSS Inc., Armonk,
NY). Dose-volume histograms were generated for all delineated clinical targets and normal tissue structures in different plans for each patient. All data were demonstrated as the medians and quartiles. A Friedman's test was used for analysis: DIBH vs. MLE $v s$. SLE. We applied posthoc Wilcoxon signed-rank test with Bonferroni correction for paired comparison. The differences in dosimetric parameters of OARs among three plans were evaluated: DIBH $v s$. MLE, DIBH $v s$. SLE, and MLE $v s$. SLE. A P value of $<0.05$ was considered statistically significant.

\section{Results}

\section{Patient characteristics}

Twenty left-sided early stage breast cancer patients were enrolled in the study. Two patients were excluded due to missing data, and another four patients were excluded due to poor image quality. Patient data of the fourteen participants were collected in this retrospective study. The median age of patients was 51 years (ranging from 39 to 70 years). All patients were female with good performance status (ECOG $=0$ ), and received breast conserving surgery (BCS) and sentinel lymph node biopsy (SLNB). In all cases, the nodes were negative including four stage 0 , seven stage IA, and three stage IIA based on the eighth edition of the AJCC. The patient characteristics are summarized in Table 1. 
Table 1 Patient and tumor characteristics ( $\mathrm{N}=14)$

\begin{tabular}{|c|c|c|}
\hline Patient characteristic & Groups & Outcomes \\
\hline Age [years] & Median [range] & 51 [39-70] \\
\hline \multirow[t]{2}{*}{ Gender, $\mathrm{n}$ [\%] } & Female & 14 [100] \\
\hline & Male & $0[0]$ \\
\hline \multirow[t]{3}{*}{ ECOG, n [\%] } & 0 & $14[100]$ \\
\hline & 1 & $0[0]$ \\
\hline & 2 & $0[0]$ \\
\hline \multirow[t]{4}{*}{ Tumor stage, $\mathrm{n}[\%]$} & Tis & $4[29]$ \\
\hline & T1 & $7[50]$ \\
\hline & T2 & $3[21]$ \\
\hline & T3-4 & $0[0]$ \\
\hline \multirow{2}{*}{ Node stage, $\mathrm{n}[\%]$} & No & 14 [100] \\
\hline & $\mathrm{N} 1-3$ & $0[0]$ \\
\hline \multirow{6}{*}{$\begin{array}{l}\text { AJCC 8th stage } \\
\text { group, } n[\%]\end{array}$} & 0 & $4[29]$ \\
\hline & IA & $7[50]$ \\
\hline & IB & $0[0]$ \\
\hline & $\| \mathrm{A}$ & $3[21]$ \\
\hline & IIB & $0[0]$ \\
\hline & III-IV & $0[0]$ \\
\hline \multirow[t]{2}{*}{ ER, n [\%] } & + & $12[86]$ \\
\hline & - & $2[14]$ \\
\hline \multirow[t]{2}{*}{$\mathrm{PR}, \mathrm{n}[\%]$} & + & $11[79]$ \\
\hline & - & $3[21]$ \\
\hline \multirow[t]{3}{*}{ HER2, n [\%] } & + & $3[21]$ \\
\hline & - & $10[71]$ \\
\hline & unknown & $1[7]$ \\
\hline
\end{tabular}

ECOG, Eastern Cooperative Oncology Group; AJCC, American Joint Committee on Cancer; T, tumor; N, node; Tis, tumor in situ; $\mathrm{n}$, number; ER, estrogen receptor; $\mathrm{PR}$, progesterone receptor; HER2, human epidermal growth factor receptor.

\section{Target volume doses}

The median volume of the CTV in the DIBH plans was $339.7 \mathrm{~cm}^{3}$ (Q1, first quartile $=260.0 \mathrm{~cm}^{3}, \mathrm{Q} 3$, third quartile $\left.=526.1 \mathrm{~cm}^{3}\right)$ and similar to other plans. Target volume doses among DIBH, MLE, and SLE plans all achieved desirable PTV_eval coverage, $95 \%$ of the prescribed dose covering at least $95 \%$ of the PTV_eval. There was no significant difference in V95\% and Dmax of PTV_eval among the three plans. Dosimetric results are summarized in Table 2.

\section{OAR doses-heart}

The mean heart dose was 4.01 Gy in SLE, 3.76 Gy in MLE, and 2.72 Gy in DIBH $(\mathrm{P}<0.001)$. DIBH plans showed significantly lower mean heart dose, $\mathrm{V} 40_{\text {heart, }}, \mathrm{V} 25_{\text {heart }}$, $\mathrm{V} 10_{\text {heart }}$ and $\mathrm{V} 5_{\text {heart }}$ compared with either MLE or SLE plans $(\mathrm{P}<0.001)$. DIBH plans could reduce the mean heart dose by $27.7 \%$ and $32.2 \%$ compared with MLE and SLE pans, respectively. DIBH plans showed a significant lower maximum heart dose than did the other plans $(\mathrm{P}=0.006)$.

DIBH plans showed significantly lower mean dose to $\mathrm{LV}, \mathrm{V} 40_{\mathrm{LV}}, \mathrm{V} 25_{\mathrm{LV}}, \mathrm{V} 10_{\mathrm{LV}}$ and $\mathrm{V} 5_{\mathrm{LV}}$ compared with other plans $(\mathrm{P}<0.001)$. DIBH plans had a significant reduction in the volume of the heart and $\mathrm{LV}$ receiving the low to high doses, which led to the lowest dose to the heart and LV, followed by MLE plans, and then SLE plans. DIBH plans had a significant lower D0.05cc of LAD compared with other plans $(\mathrm{P}=0.017)$. DIBH plans showed a significant reduction in the mean LAD dose $(\mathrm{P}<0.001): 20.6 \%$ less than MLE plans, $25.3 \%$ less than SLE plans. There was no significantly difference between MLE and SLE plans for the mean dose to LAD.

\section{OAR doses-lung}

The V20 left lung was $16.25 \%$ in SLE, $15.79 \%$ in MLE and $14.20 \%$ in DIBH $(\mathrm{P}<0.001)$. DIBH plans showed significantly lower left lung mean dose, $\mathrm{V} 40_{\text {left lung }}, \mathrm{V} 30_{\text {left lung, }}$, $\mathrm{V} 20_{\text {left lung, }}, \mathrm{V} 10_{\text {left lung }}$ and $\mathrm{V} 5_{\text {left lung }}$ when compared with either MLE or SLE plans. For the low-high dose regions in the left lung, there was no significant difference between MLE and SLE plans.

The right lung mean doses were very low in all three plans. There was no significant difference in right lung mean dose $(\mathrm{P}=0.211)$, and $\mathrm{V} 5_{\text {right lung }}(\mathrm{P}=0.281)$ among three plans.

Figure 4 shows the isodose distributions for DIBH, MLE, and SLE plans on axial images from a case of this study. The average DVHs of heart, LV, LAD and left lung of three plans are shown in Figure 5.

\section{Discussion}

The incidence of female breast cancer in Asian Countries has increased rapidly over the past 40 years. Rapidly increasing incidence of breast cancer in East Asia has primarily been attributed to genetic and environmental influences, including westernization $(2,19)$. In Taiwan, 
Table 2 Dosimetric results for PTV_eval and organs at risk in left side breast cancer and quick reference guide among DIBH, MLE and SLE

\begin{tabular}{|c|c|c|c|c|c|c|c|}
\hline & $\begin{array}{l}\text { DIBH, median } \\
\quad(\mathrm{Q} 1, \mathrm{Q} 3)\end{array}$ & $\begin{array}{l}\text { MLE, median } \\
\text { (Q1, Q3) }\end{array}$ & $\begin{array}{l}\text { SLE, median } \\
\text { (Q1, Q3) }\end{array}$ & $P$ value & \multicolumn{3}{|c|}{ Quick reference guide (P value) } \\
\hline \multicolumn{8}{|l|}{ PTV_eval } \\
\hline V95\% & 98.50 (97.68-98.97) & $98.17(96.80-99.20)$ & $98.06(97.30-99.08)$ & 0.382 & & NA & \\
\hline \multicolumn{8}{|l|}{ Heart } \\
\hline${ }^{*} \operatorname{Dmax}(\mathrm{Gy})$ & $50.50(44.95-51.75)$ & $50.70(48.75-51.55)$ & $52.10(50.45-53.50)$ & ${ }^{*} 0.006$ & - & $\mathrm{DIBH}$ & - \\
\hline *Dmean (Gy) & $2.72(2.05-3.32)$ & $3.76(2.34-4.16)$ & $4.01(3.03-4.69)$ & ${ }^{*}<0.001$ & $\mathrm{DIBH}$ & $\mathrm{DIBH}$ & MLE \\
\hline *V40 & $0.65(0.12-1.34)$ & $1.75(0.33-2.28)$ & $2.32(0.87-3.22)$ & ${ }^{*}<0.001$ & $\mathrm{DIBH}$ & $\mathrm{DIBH}$ & MLE \\
\hline *V5 & $8.10(5.91-10.36)$ & $11.75(6.69-12.71)$ & $12.86(10.17-13.95)$ & ${ }^{*}<0.001$ & $\mathrm{DIBH}$ & $\mathrm{DIBH}$ & MLE \\
\hline \multicolumn{8}{|l|}{ LV } \\
\hline *Dmean (Gy) & $3.66(2.37-4.28)$ & $4.69(3.06-5.75)$ & $5.62(3.97-6.95)$ & ${ }^{*}<0.001$ & $\mathrm{DIBH}$ & $\mathrm{DIBH}$ & MLE \\
\hline *V40 & $0.75(0.02-1.62)$ & $1.41(0.10-3.70)$ & $3.01(0.84-5.51)$ & ${ }^{*}<0.001$ & $\mathrm{DIBH}$ & $\mathrm{DIBH}$ & MLE \\
\hline *V25 & $2.91(0.52-4.65)$ & 4.88 (1.68-7.95) & $6.91(3.19-9.96)$ & ${ }^{*}<0.001$ & $\mathrm{DIBH}$ & $\mathrm{DIBH}$ & MLE \\
\hline *V10 & $6.96(3.47-9.48)$ & $10.25(5.67-13.54)$ & $12.72(7.90-16.23)$ & ${ }^{*}<0.001$ & $\mathrm{DIBH}$ & $\mathrm{DIBH}$ & MLE \\
\hline *V5 & $11.53(7.64-13.78)$ & $15.42(10.00-18.21)$ & $18.97(12.98-22.21)$ & ${ }^{*}<0.001$ & DIBH & $\mathrm{DIBH}$ & MLE \\
\hline *Dmean (Gy) & $7.63(7.04-8.57)$ & $8.32(7.85-9.90)$ & $8.64(7.91-10.59)$ & ${ }^{*}<0.001$ & $\mathrm{DIBH}$ & $\mathrm{DIBH}$ & - \\
\hline *V40 & $7.23(5.86-9.13)$ & $8.07(7.41-12.26)$ & $8.53(7.80-12.58)$ & ${ }^{*}<0.001$ & $\mathrm{DIBH}$ & $\mathrm{DIBH}$ & - \\
\hline *V30 & $10.75(9.58-12.67)$ & $12.08(11.10-15.74)$ & $12.55(11.30-16.73)$ & ${ }^{*}<0.001$ & $\mathrm{DIBH}$ & DIBH & - \\
\hline *V20 & $14.20(13.27-16.51)$ & $15.79(14.65-19.12)$ & $16.25(14.69-20.33)$ & ${ }^{*}<0.001$ & $\mathrm{DIBH}$ & $\mathrm{DIBH}$ & - \\
\hline${ }^{*} \mathrm{~V} 10$ & $20.16(18.77-22.24)$ & $21.62(20.53-24.35)$ & $22.08(20.34-25.68)$ & ${ }^{*} 0.005$ & $\mathrm{DIBH}$ & $\mathrm{DIBH}$ & - \\
\hline *V5 & $26.62(25.38-28.63)$ & $28.35(27.44-30.41)$ & $28.49(27.67-31.78)$ & *0.005 & $\mathrm{DIBH}$ & $\mathrm{DIBH}$ & - \\
\hline \multicolumn{8}{|l|}{ Right lung } \\
\hline Dmean (Gy) & $0.32(0.29-0.38)$ & $0.37(0.31-0.40)$ & $0.35(0.31-0.40)$ & 0.211 & & NA & \\
\hline V5 & $0.08(0.00-0.14)$ & $0.02(0.00-0.11)$ & $0.01(0.00-0.07)$ & 0.281 & & NA & \\
\hline
\end{tabular}

*, significant $P$ value $(P<0.05)$; ", quick reference guide is based on the significant $P$ value $(P<0.05)$ of post-hoc test, "-" means statistical insignificance. PTV_eval, created from the PTV cropped $3 \mathrm{~mm}$ from the skin surface; DIBH, deep inspiration breath hold; MLE, middle of lung expansion; SLE, start of lung expansion; NA, not applicable; Q1, first quartile; Q3, third quartile; Dmax , maximum dose; Dmean, mean dose; $V x \%$, the percent volume receiving at least $x \%$ of the prescribed dose ; $V x$, the percent volume receiving at least $X G y$. 


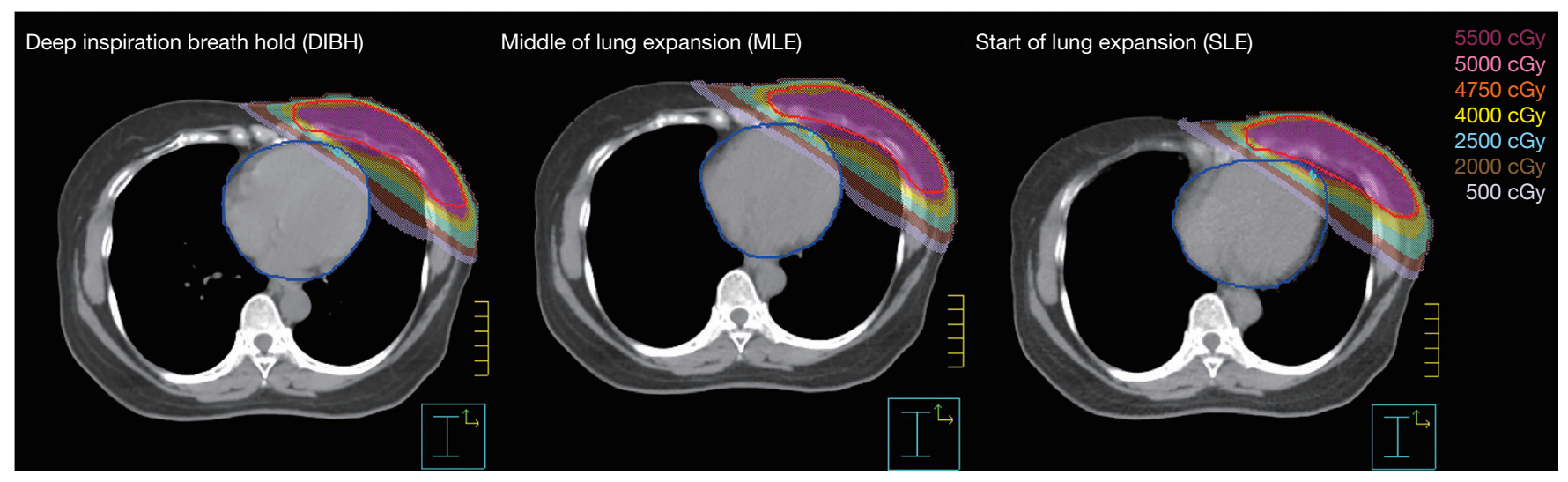

Figure 4 Isodose distributions for DIBH, MLE, and SLE plans on axial images from a case of this study. The slices are taken at 12 slices below mid-breast level. Contouring of PTV_eval (red line), heart (dark blue line) and LAD (blue line) were shown. DIBH, deep inspiration breath hold; MLE, middle of lung expansion; SLE, start of lung expansion; PTV_eval, created from the PTV cropped 3 mm from the skin surface; LAD, left anterior descending artery.

breast cancer is the most common cancer in women, and the incidence of breast cancer increased over time among female aged 40-75 years. The risk of breast cancer recurrence exists after treatment even after 10 years. Whole breast irradiation after BCS reduces local recurrence rate and benefits patient on survival.

However, concerns have been raised about the risk of acute and chronic radiation induced side effects, especially when breast cancer happen in young age and most patients could have long survival compared to other malignancies.

The primary goal of radiotherapy is to achieve homogeneous dose distribution throughout the target volume and careful attention must be paid to the amount of lung tissue and heart involved in the treatment field. Gagliardi et al. have related Heart V25 Gy to cardiac mortality (20) and a correlation between the percent of the $L V$ within the radiation field and percent of patients with perfusion defects on cardiac Single Photon Emission Computed Tomography (SPECT) was found (21). Surprisingly, the data suggested that there was no apparent threshold for cardiac exposure necessary to cause a perfusion defect. The potential harmful cardiac effect from RT can be amplified by the use of anthracycline-based chemotherapy, trastuzumab and aromatase inhibitors. Increased risk of cardiac disease was noted for patients with underlying risk factors of coronary artery disease receiving radiotherapy (3).

With deep inspiration, the heart structure moves inferiorly and posteriorly, away from the deep border of the tangential field and resulting in significant dose reduction. Since there are no safety threshold doses for the heart,
$\mathrm{LV}$ or $\mathrm{LAD}$, it is crucial to minimize radiation exposure as low as achievable. Studies have demonstrated that DIBH is a technique that takes advantage of a more favorable position of the OARs during inspiration to minimize doses of normal organs over a course of radiation therapy (10). Consideration in applying a DIBH technique using $\mathrm{ABC}$ for left-sided breast RT is variability in organ and structure position, and which draws our attention due to a marked anatomic shift during respiratory cycle.

Our study presented a dosimetric comparison of adjuvant left-sided breast cancer radiotherapy with DIBH technique among different respiratory phases. Fourteen left breast cancer patients received adjuvant left breast radiotherapy after breast-sparing surgery were analyzed in our study. Different from most studies which compared FB with DIBH using 3D-CT images for dosage analysis, we used 4D-CT image during deep respiration for comparison in order to elucidate dosimetric benefit of DIBH technique more precisely between different lung expansion phases. We obtained satisfactory PTV_eval coverage and no significant difference was noted among the three plans.

We reported that the use of $\mathrm{DIBH}$ technique could provide superior dose sparing for heart, LV, LAD, and left lung, which is consistent with previous studies (10,22). The mean heart dose was 2.72 Gy in DIBH plans which reduced the mean heart dose by $27.7 \%$ and $32.2 \%$ compared with MLE and SLE pans, respectively. We also found that through inhalation cycle, the irradiated dose to heart and LV gradually decreased, and our results indicated that DIBH was superior over other plans in terms of OAR 

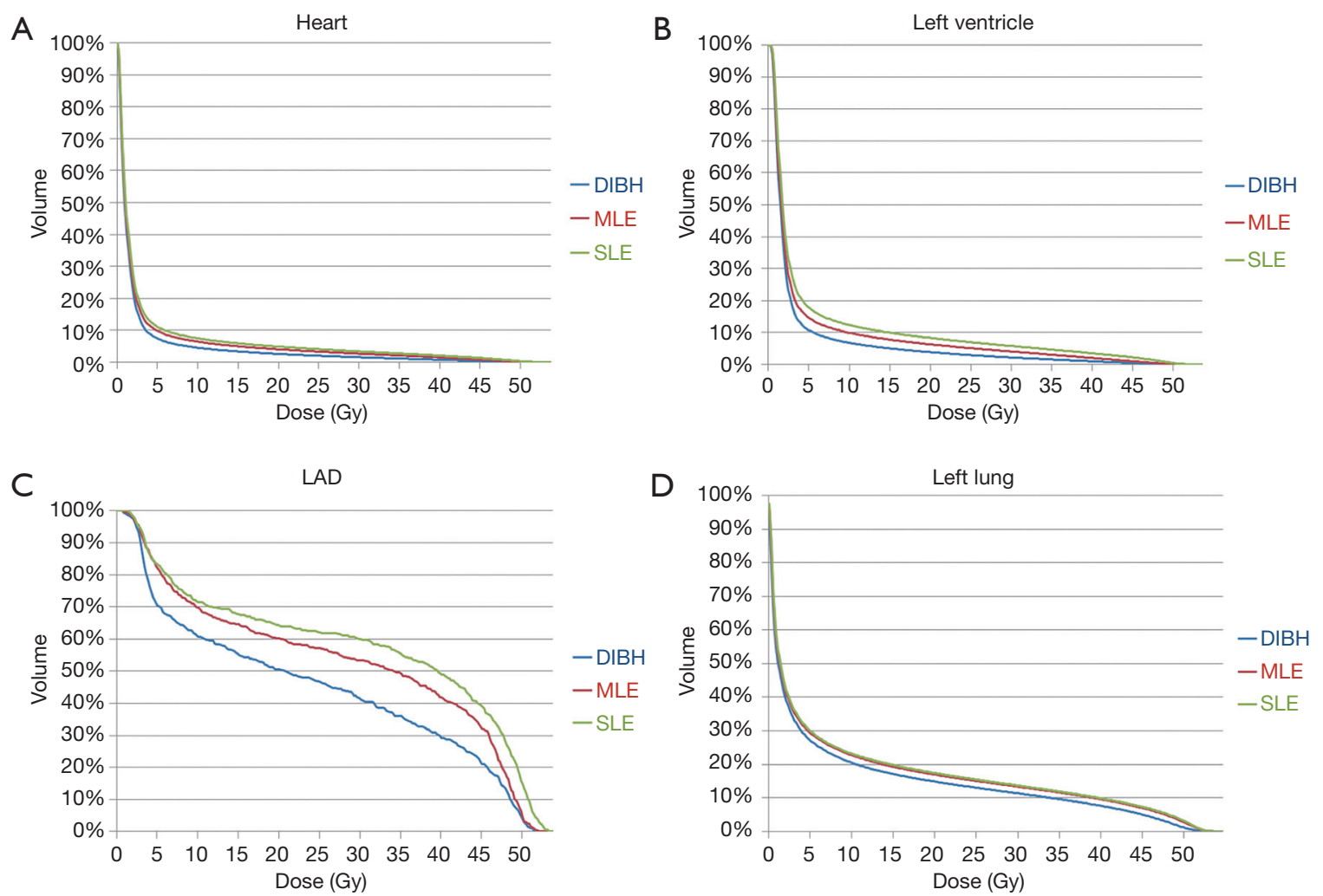

Figure 5 The dose-volume histograms (DVHs) of DIBH, MLE, and SLE plans in (A) heart, (B) LV, (C) LAD, and (D) left lung. DIBH, deep inspiration breath hold; MLE, middle of lung expansion; SLE, start of lung expansion; LV: left ventricle; LAD, left anterior descending artery.

sparing, followed by MLE, and then SLE.

Ledsom et al. reported that a positive correlation was identified between DIBH amplitude and mean cardiac dose reduction (23). In our study, we found that DIBH plans showed significantly lower D $0.05 \mathrm{cc}$ of LAD and Dmax of heart compared with SLE plans, but there was no significant difference between DIBH and MLE plans, and MLE and SLE plans, which implied that the extremely high dose region could be spared only with enough inspiration. We also found that DIBH plans showed a significant reduction in the mean LAD dose compared with MLE (less than $20.6 \%$ ) and SLE plans (less than 25.3\%).

We obtained significantly lower left lung mean dose, including V40 V30, V20, V10 and V5 in DIBH plans compared with both MLE and SLE plans. These observations are consistent with those from previous studies that used DIBH strategies $(22,24)$. By contrast, Comsa et al. found no statistically significant difference in the left lung mean dose, and noted a statistically significant increase in left lung V5 with the DIBH technique (25). In our study, there was no significantly difference between MLE and SLE plans in the low-high dose regions of left lung. There was no significant difference in right lung mean dose and V5 among three plans, which could be expected probably due to the extremely low dose exposure to contralateral lung during radiotherapy.

One of the limitations of our study is that we did not use intravenous contrast for the planning CT scans, and this might reduce the accuracy in contouring the LAD. Though, we could still visualize the LAD structure on most of the CT slices directly. In addition, the LAD moves with cardiac motion, and contouring uncertainties might be present, and thus we chose to measure the D0.05 cc of the LAD rather the maximum point. Another limitation is the relatively obscured CT images retrieval from 4D-CT due to reorganizing images during respiratory motion. The other limitation of the study also includes small patient number, thus there might be a selection bias and the result may not be applicable to all the patients, such as smaller or bigger breasts. However, the range of CTV size was still wide in 
our study $\left(\mathrm{Q} 1=260.0 \mathrm{~cm}^{3}, \mathrm{Q} 3=526.1 \mathrm{~cm}^{3}\right)$.

$\mathrm{DIBH}$ technique with $\mathrm{ABC}$ device is a mature technology in our department with well-trained and experienced medical staff. It usually takes approximately 5 to 10 minutes more in each treatment for patient using DIBH technique compared with FB. Further study with accuracy assessment for respiratory motion and respiration threshold is needed to further validate the clinical use of DIBH technique in breast cancer radiation treatment planning.

In summary, our study compared dosage for the adjuvant treatment of left-sided breast cancer using DIBH technique with different respiratory phases based on 4D-CT images. We reported that the use of $\mathrm{DIBH}$ technique resulted in a significant dose reduction in heart, LV, LAD, and left lung, especially with enough deep inspiration. Hence, the DIBH technique could be considered as a promising tool for normal organ sparing. Further study is required to validate the clinical benefit of DIBH technique for patients with left breast cancer and establish a relationship between DIBH amplitude and normal organ dose reduction.

\section{Acknowledgments}

Funding: The authors disclosed receipt of the following financial support for the research and publication of this article: This work was supported by Taipei Medical University - Shuang-Ho Hospital (grant number: 109SHHR-06).

\section{Footnote}

Reporting Checklist: The authors have completed the MDAR reporting checklist. Available at http://dx.doi.org/10.21037/ tro-20-49

Conflicts of Interest: All authors have completed the ICMJE uniform disclosure form (available at http://dx.doi. org/10.21037/tro-20-49). All authors report grants from Taipei Medical University - Shuang-Ho Hospital, outside the submitted work.

Ethical Statement: The authors are accountable for all aspects of the work in ensuring that questions related to the accuracy or integrity of any part of the work are appropriately investigated and resolved. The study was conducted in accordance with the Declaration of Helsinki (as revised in 2013). This study is retrospective, and approved by the hospital IRB (TMU-JIRB No.: N201912026) as
"Expedited Review"; therefore, the informed consent is not required.

Open Access Statement: This is an Open Access article distributed in accordance with the Creative Commons Attribution-NonCommercial-NoDerivs 4.0 International License (CC BY-NC-ND 4.0), which permits the noncommercial replication and distribution of the article with the strict proviso that no changes or edits are made and the original work is properly cited (including links to both the formal publication through the relevant DOI and the license). See: https://creativecommons.org/licenses/by-nc-nd/4.0/.

\section{References}

1. Bray F, Ferlay J, Soerjomataram I, et al. Global cancer statistics 2018: GLOBOCAN estimates of incidence and mortality worldwide for 36 cancers in 185 countries. CA Cancer J Clin 2018;68:394-424.

2. Gomez SL, Yao S, Kushi LH, et al. Is Breast Cancer in Asian and Asian American Women a Different Disease? J Natl Cancer Inst 2019;111:1243-4.

3. Waks AG, Winer EP. Breast Cancer Treatment: A Review. JAMA 2019;321:288-300.

4. Darby S, McGale P, Correa C, et al. Effect of radiotherapy after breast-conserving surgery on 10-year recurrence and 15-year breast cancer death: meta-analysis of individual patient data for 10,801 women in 17 randomised trials. Lancet 2011;378:1707-16.

5. Meattini I, Guenzi M, Fozza A, et al. Overview on cardiac, pulmonary and cutaneous toxicity in patients treated with adjuvant radiotherapy for breast cancer. Breast Cancer 2017;24:52-62.

6. Darby SC, Ewertz M, McGale P, et al. Risk of ischemic heart disease in women after radiotherapy for breast cancer. N Engl J Med 2013;368:987-98.

7. Drost L, Yee C, Lam H, et al. A Systematic Review of Heart Dose in Breast Radiotherapy. Clin Breast Cancer 2018;18:e819-24.

8. Jeba J, Isiah R, Subhashini J, Backianathan S, Thangakunam B, Christopher DJ: Radiation Pneumonitis After Conventional Radiotherapy For Breast Cancer: A Prospective Study. J Clin Diagn Res 2015;9:XC01-5.

9. Vasiljevic D, Arnold C, Neuman D, et al. Occurrence of pneumonitis following radiotherapy of breast cancer - A prospective study. Strahlenther Onkol 2018;194:520-32.

10. Bergom C, Currey A, Desai N, et al. Deep Inspiration Breath Hold: Techniques and Advantages for Cardiac 
Sparing During Breast Cancer Irradiation. Front Oncol 2018;8:87.

11. Keall PJ, Vedam SS, George R, et al. Respiratory regularity gated 4D CT acquisition: concepts and proof of principle. Australas Phys Eng Sci Med 2007;30:211-20.

12. Huang TC, Wang YC, Kao CH. Thoracic tumor volume delineation in 4D-PET/CT by low dose interpolated CT for attenuation correction. PLoS One 2013; 8: e75903.

13. Thomas SJ, Hoole AC: The effect of optimization on surface dose in intensity modulated radiotherapy (IMRT). Phys Med Biol 2004;49:4919-28.

14. Ashburner MJ, Tudor S: The optimization of superficial planning target volumes (PTVs) with helical tomotherapy. J Appl Clin Med Phys 2014; 15: 4560.

15. Sankar A, Velmurugan J: Different intensity extension methods and their impact on entrance dose in breast radiotherapy: A study. J Med Phys 2009;34:200-5.

16. Tyran M, Tallet A, Resbeut M, et al.: Safety and benefit of using a virtual bolus during treatment planning for breast cancer treated with arc therapy. J Appl Clin Med Phys 2018;19:463-72.

17. Feng M, Moran JM, Koelling T, et al. Development and validation of a heart atlas to study cardiac exposure to radiation following treatment for breast cancer. Int J Radiat Oncol Biol Phys 2011;79:10-8.

18. Marks LB, Yorke ED, Jackson A, et al. Use of normal tissue complication probability models in the clinic. Int $\mathrm{J}$

doi: $10.21037 /$ tro-20-49

Cite this article as: Kuo CC, Chang CC, Cheng HW, Tsai JT. Impact of Active Breathing Control-Deep Inspiration Breath Hold (ABC-DIBH) on the dose to surrounding normal structures in tangential field left breast radiotherapy. Ther Radiol Oncol 2020;4:26.
Radiat Oncol Biol Phys 2010;76:S10-9.

19. Lin CH, Yap YS, Lee KH, et al. Contrasting Epidemiology and Clinicopathology of Female Breast Cancer in Asians vs the US Population. J Natl Cancer Inst 2019;111:1298-306.

20. Gagliardi G, Constine LS, Moiseenko V, et al. Radiation dose-volume effects in the heart. Int J Radiat Oncol Biol Phys 2010;76:S77-85.

21. Kaidar-Person O, Zagar TM, Oldan JD, et al. Early cardiac perfusion defects after left-sided radiation therapy for breast cancer: is there a volume response? Breast Cancer Res Treat 2017;164:253-262.

22. Lai J, Hu S, Luo Y, et al. Meta-analysis of deep inspiration breath hold (DIBH) versus free breathing (FB) in postoperative radiotherapy for left-side breast cancer. Breast Cancer 2020;27:299-307.

23. Ledsom D, Reilly AJ, Probst H: Assessment of deep inspiration breath hold (DIBH) amplitude and reduction in cardiac dose in left breast cancer patients. Radiography (Lond) 2018;24:98-103.

24. Shim JG, Kim JK, Park W, et al. Dose-Volume Analysis of Lung and Heart according to Respiration in Breast Cancer Patients Treated with Breast Conserving Surgery. J Breast Cancer 2012;15:105-110.

25. Comsa D, Barnett E, Le K, et al. Introduction of moderate deep inspiration breath hold for radiation therapy of left breast: Initial experience of a regional cancer center. Pract Radiat Oncol 2014;4:298-305. 